Referências

1. Associação Brasileira de Psiquiatria. Diretrizes para a indústria da moda - Recomendações da Comissão Técnica Brasileira de Grupos Especializados no Estudo e Tratamento de Transtornos Alimentares. 2007 [cited May 12 2007]; Available from: URL: http:// www.abpbrasil.org.br/newsletter/comissao_ta/diretrizes_moda.pdf

2. Moya T, Claudino AM, van Furth EF. Extreme thinness in models mobilizes eating disorders' researchers and specialists. Rev Bras Psiquiatr. 2007;29(1):1-2.

3. Pinheiro AP, Sullivan PF, Bacaltchuck J; do Prado-Lima PA, Bulik CM. Genética em transtornos alimentares: ampliando os horizontes de pesquisa. Rev Bras Psiquiatr. 2006;28(3):218-25.

4. Polivy J, Herman CP. Causes of eating disorders. Annu Rev Psychol. 2002; 53:187-213.

5. Rodrigues AM, Cintra IP, Fisberg M. Perfil nutricional, composição corporal e hábitos alimentares de modelos adolescentes. Pediatria Moderna. 2005;41(4):170-8.

\section{Acerca do documento da Comissão Técnica da ABP intitulado "Diretrizes para a Indústria da Moda"}

\author{
Regarding the ABP Technical \\ Commission's document entitled \\ "Fashion Industry Guidelines'"
}

\section{Sr. Editor,}

Os autores ressaltaram, em seu editorial (Moya et al., 2007), que os transtornos alimentares têm sido compreendidos dentro de um modelo de etiologia multifatorial; assim, a magreza, enquanto "padrão de beleza", pode representar apenas "um fator" dentre a complexa rede de fatores de risco para a gênese e manutenção dos transtornos alimentares. ${ }^{1}$

De fato, a maioria dos estudos que avaliam a influência da mídia na insatisfação com o corpo disponível até o presente momento são do tipo transversal, e, portanto, não podem ser utilizados de forma incontestável na determinação de relações de causalidade. No entanto, alguns estudos prospectivos iniciais, ${ }^{2,3}$ salvaguardadas suas limitações, também sugerem a existência de possível associação entre a exposição à mídia e o desenvolvimento de atitudes e comportamentos alimentares e de controle de peso inadequados, assim como a insatisfação corporal e o desejo de mudança física em adolescentes, aspectos considerados potenciais para o desenvolvimento de transtorno alimentar. Em seu estudo, Becker et al. apontam o aumento significante de tais comportamentos após a introdução da televisão nas Ilhas Fiji. ${ }^{3}$

A anorexia nervosa é uma doença relativamente rara, o que dificulta muito a realização de estudos longitudinais com desenho ideal para a determinação de seus fatores de risco causais. Tendo em vista seu alto índice de mortalidade, bem como o potencial risco de cronificação e altos custos de tratamento, a Comissão Técnica da Associação Brasileira de Psiquiatria (ABP) considera prudente a adoção de medidas preventivas, ainda que não conte com fortes evidências de eficácia. Isso já é feito no tratamento da anorexia nervosa, que é oferecido apesar das principais diretrizes para seu tratamento ainda não estarem embasadas por evidências de bom nível e, por vezes, pautadas em consenso de experts. ${ }^{4}$ Embora não conste das recomendações da Comissão Técnica, ela tem como objetivo avaliar ações preventivas por meio de projetos de pesquisa. Corroborando esta iniciativa, existe o fato de que pesquisadores da área têm realizado inúmeras intervenções preventivas que envolvem, inclusive, a abordagem da insatisfação corporal. ${ }^{1,5}$ É certo que estas ações devem ser aprimoradas e reavaliadas ao longo do tempo, à medida que evidências científicas mais sólidas surjam.

A fundamentação para a escolha do limite de $18,5 \mathrm{~kg} / \mathrm{m}^{2}$ de IMC baseia-se nos parâmetros que a Organização Mundial de Saúde (OMS) estabelece como "peso mínimo saudável para a população geral". No que tange à recomendação para se evitar o uso de modelos com IMC abaixo de $18,5 \mathrm{k} / \mathrm{m}^{2}$, busca-se: 1) diminuir a pressão sobre as modelos para emagrecer e, assim, garantir sua saúde e segurança no ambiente profissional; 2) veicular padrões físicos concebidos como mais saudáveis e mais próximos da realidade da maioria da população exposta à mídia. A sugestão de diversificação de biotipos relaciona-se, portanto, à "redução de estigma" tanto no exercício da profissão de modelagem quanto na sociedade de maneira geral.

Sabe-se que o médico detém, em função da sua profissão, a responsabilidade de cuidar da saúde dos indivíduos, inclusive tomando ações preventivas e não apenas de tratamento. A cultura é um processo dinâmico, em constante transformação, resultante da atuação de todos os indivíduos da sociedade. Entendemos que o médico, como ser social e membro integrante deste grupo, é parte ativa e atuante, e tem, portanto, toda a legitimidade para estimular transformações culturais voltadas para a adoção de padrões e comportamentos que auxiliem na promoção de saúde.

Tatiana Moya Grupo de Obesidade e Transtornos Alimentares, Instituto Estadual de Diabetes e Endocrinologia, Instituto de Psiquiatria da Universidade do Brasil, Universidade Federal do Rio de Janeiro (GOTA - IEDE - IPUB/UFRJ),

Rio de Janeiro (RJ), Brasil

Angélica M Claudino Programa de Orientação e Assistência aos Transtornos Alimentares, Escola Paulista de Medicina, Universidade Federal de São Paulo (PROATA - UNIFESP/EPM), São Paulo (SP), Brasil

Eric F van Furth Center for Eating Disorders Ursula, Leidschendam, the Netherlands

Department of Psychiatry, Leiden University, the Netherlands

Financing: None

Conflict of interests: None

\section{Referências}

1. Levine MP, Piran N. The role of body image in prevention of eating disorders. Body Image. 2004;1(1):57-70.

2. Field AE, Camargo CA, Taylor CB, Berkey CS, Colditz GA. Relation of peer and media influences to the development of purging behaviors among preadolescent and adolescent girls. Arch Pediatr Adolesc Med. 1999;153(11):1184-9.

3. Becker AE, Burwell RA, Gilman SE, Herzog DB, Hamburg P. Eating behaviours and attitudes following prolonged exposure to television among ethnic Fijian adolescent girls. $\mathrm{Br} J$ Psychiatr. 2002;180:509-14. 
4. American Psychiatric Association. Practice guideline for the treatment of patients with eating disorders. 3rd ed. [128 screens]. [updated 2006 May] [cited 2007 Jun 17]. Available from: http:// www.psych.org/psych_pract/treatg/pg/EatingDisorders3ePG_0428-06.pdf

5. Levine MP, Smolak L. Prevention of negative body image, disordered eating, and eating disorders: an update. In: Wonderlich S, Mitchell JE, Zwaan M, Steiger H, editors. Annual Review of Eating Disorders. United Kingdon: Radcliffe Publishing; 2007. p. 1-13.

\section{Observações sobre 0 artigo "Subtipos clínicos do transtorno obsessivo-compulsivo com base na presença de compulsões de checagem e lavagem" de Fontenelle et al. (2005)}

Remarks on the paper" "Clinical subtypes of obsessive-compulsive disorder based on the presence of checking and washing compulsions" by Fontenelle et al. (2005)

\section{Sr. Editor,}

Pesquisas apontam para o caráter heterogêneo do transtorno obsessivo-compulsivo (TOC). As diferenças nas taxas de resposta ao tratamento, nos cursos evolutivos e nas várias formas de apresentação da síndrome sugerem a existência de diversos subtipos com possíveis bases fisiopatológicas específicas.

No intuito de estudar O TOC, seja do ponto de vista fenomenológico ou genético, torna-se fundamental a caracterização de fenótipos clínicos mais precisos. A identificação de subgrupos mais homogêneos é uma etapa importante na identificação de mecanismos fisiopatológicos e no desenvolvimento de estratégias terapêuticas eficazes.

Compulsões de verificação e de lavagem são as mais freqüentes em amostras clínicas. ${ }^{1}$ Embora muitos pacientes apresentem ambas as dimensões de forma simultânea, geralmente uma predomina, o que permite classificá-los como "verificadores" ou "lavadores".

Apesar do interesse crescente na identificação de grupos mais homogêneos de pacientes, poucos autores verificaram se lavagem e verificação são possíveis marcadores de subtipos do transtorno. ${ }^{2}$

Nessa linha de investigação, Fontenelle et al. utilizaram uma metodologia original e alocaram 106 portadores de TOC em quatro subgrupos: o primeiro, composto pelos verificadores; o segundo, pelos lavadores; o terceiro, pelos que apresentavam lavagem e verificação simultaneamente; e o último, composto pelos que não apresentavam nenhum dos dois comportamentos compulsivos. ${ }^{2}$

No entanto, gostaríamos de tecer algumas considerações acerca da metodologia. Inicialmente, a descrição do percentual de pacientes do estudo naturalístico realizado pelos autores que participaram do atual poderia auxiliar na melhor compreensão do desenho do estudo realizado. ${ }^{3}$

No caso de não terem sido incluídos todos os pacientes do estudo naturalístico, seria possível que tenha contribuído para um viés de seleção? Será que pacientes que, por alguma razão, não tenham sido incluídos poderiam ser portadores de algum subtipo específico, influenciando na descrição dos resultados?

Quanto aos dados levantados durante a investigação, cumpre indagar: foram colhidas informações sobre a história familiar dos subtipos? Seria a história familiar importante no desenvolvimento dos mesmos?

Se considerarmos, ainda, que a amostra foi retirada de um estudo naturalístico, talvez os autores pudessem ter apresentado dados acerca da resposta farmacológica entre os subtipos, o que aumentaria a importância do trabalho.

Apesar das considerações, sem dúvida o estudo de Fontenelle et al. fornece dados e idéias para estudos e investigações posteriores com amostras maiores de pacientes. A identificação de subtipos específicos talvez possa diminuir a tão descrita "heterogeneidade" do TOC e, assim, contribuir para delinear subgrupos mais homogêneos desse transtorno.

Embora com número crescente de trabalhos científicos, o TOC continua um desafio para clínicos e pesquisadores. Apesar do surgimento de novas abordagens terapêuticas, $40-60 \%$ dos pacientes não respondem ao tratamento psicofarmacológico; mais de $70 \%$ dos clínicos e pesquisadores consideram a Terapia de Exposição e Prevenção de Respostas (EPR) efetiva; porém, ela não demonstra eficácia cientificamente comprovada para metade dos que começam o tratamento e para $25 \%$ dos que a terminam. ${ }^{4,5}$

A investigação do TOC em subtipos pode ter implicações para o diagnóstico, avaliação clínica, padrão de comorbidade, mecanismos fisiopatológicos subjacentes e melhora na formulação e na predição de resposta ao tratamento. Acreditamos que só assim será possível desenvolver intervenções terapêuticas mais precisas e eficazes para um maior número de portadores.

Luciana Nagalli Gropo, Anna Guerra F Lima Toniolo, Elenita Domingues da Silva, Ilduara Valéria Sidrim Figueiredo, Kátia Petribú Consórcio Nacional de Pesquisa sobre Transtornos do Espectro Obsessivo-Compulsivo - Núcleo de Pernambuco (C-TOC-PE), Recife (PE), Brasil

Hospital Universitário Oswaldo Cruz, Faculdade de Ciências Médicas, Universidade de Pernambuco (UFPE), Recife (PE), Brasil

Financiamento: Inexistente

Conflito de interesse: Inexistente

\section{Referências}

1. Fontenelle LF, Mendlowicz MV, Marques C, Versiani M. Trans-cultural aspects of obsessive-compulsive disorder: a description of a Brazilian sample and a systematic review of international clinical studies. J Psychiatr Res. 2004;38(4):403-11.

2. Fontenelle LF, Mendlowicz MV, Versiani M. Clinical subtypes of obsessive-compulsive disorder based on the presence of checking and washing compulsions. Rev Bras Psiquiatr. 2005;27(3):201-7.

3. Pereira MG. Epidemiologia. Teoria e Prática. Rio de Janeiro: Guanabara Koogan; 1999. 